\title{
High-grade leiomyosarcoma of the transverse colon presenting with bowel perforation
}

\author{
Efstratios Koutroumpakis, MD, ${ }^{a}$ Hussein Assi, MD, ${ }^{a}$ Lezah McCarthy, MD, ${ }^{\mathrm{b}}$ and Syed \\ Mehdi, MDc
}

${ }^{a}$ Department of Internal Medicine, Albany Medical Center, Albany, New York; and ${ }^{\mathrm{b}}$ Department of Pathology and ${ }^{\mathrm{c}}$ Division of Hematology-Oncology, Department of Internal Medicine, Stratton Veterans Affairs Medical Center, Albany, New York

$\mathrm{S}$ tromal or mesenchymal tumors account for about $1 \%$ of gastrointestinal (GI) tract neoplasms and are divided into 2 main categories: the gastrointestinal stromal tumors (GISTs; 60\%$90 \%$ of mesencymal tumors), and the non-GIST neoplasms (10\%-30\% of mesencymal tumors). ${ }^{1}$ The nonGIST neoplasms consist of a heterogenous group of soft-tissue tumors, identical to soft-tissue tumors elsewhere in the body. These tumors include lipomas, liposarcomas, leiomyomas, leiomyosarcomas, desmoid tumors, schwannomas, and peripheral nerve sheath tumors. GIST tumors are distinguished from nonGIST based on immunohistochemical staining positive for CD34, KIT, DOG1, or less commonly based on genetic analysis showing activating mutations in the KIT or PDGFRA gene..$^{1}$ Leiomyosarcomas of the GI tract are rare neoplasms and usually involve the small intestine and colon. ${ }^{1}$ Many of the mesenchymal tumors reported as leiomyosarcomas in the preGIST era are now classified as GIST. ${ }^{2}$ Differentiation between the 2 is critical because of the differences in management and prognosis.

\section{Case presentation and summary}

A 68-year-old white woman presented to the emergency department of a local hospital with severe diffuse abdominal pain and nausea that had worsened over the previous 2 weeks. She reported no changes in her bowel movements. Her medical history was not significant, but her surgical history included an anterior chest wall lipoma excision and a total abdominal hysterectomy with bilateral salpingo-oophorectomy for postmenopausal dysfunctional uterine bleeding secondary to multiple uterine leiomyomata. Both procedures had been performed 15 years previously. The patient reported no family history of cancer or current or past use of tobacco, alcohol, or recreational drugs.

A physical examination revealed diffuse, direct, and rebound abdominal tenderness, and guarding, and the results of the laboratory tests including a complete blood count and a comprehensive metabolic profile were significant only for a leukocytosis level of 13,600 cells $/ \mathrm{mm}^{3}$ (normal range 4,000 9,000 cells $/ \mathrm{mm}^{3}$ ). All the rest of the lab values were within normal limits. A computed-tomography (CT) scan of the abdomen and pelvis showed free abdominal air, suggestive of perforated viscous, as well as a large mass at the level of transverse colon. The patient was admitted for an exploratory laparotomy and was found to have a transverse colon mass that was grossly perforating the colonic wall. Segmental resection of the transverse colon and right-sided end colostomy was subsequently performed using the Hartmann procedure. On gross examination, the mass was $11.5 \times 9.5 \times 9 \mathrm{~cm}$ and was invading and perforating the colonic wall.

Microscopically, the tumor's mitotic rate was $17 / 10$ high-power fields. Marked pleomorphism and necrosis (less than 10\%) were noted. In immunohistochemical testing, tumor cells stained positive for desmin and actin, but did not stain for pan-keratin, CD117, s-100, or CD34. The aforementioned findings were suggestive of high-grade leiomyosarcoma (Figures 1, 2 , and 3 on p. 362). In addition, pathology showed positive surgical margins, and 2 out of the 14 resected lymph nodes were positive for metastatic disease. A CT scan of the chest, abdomen, and pelvis did not show any distant metastases, and the stage of the disease was considered to be T2bN1M0.

The treatment plan was for further surgical man-

Accepted for publication November 11, 2015. Correspondence: Efstratios Koutroumpakis, MD; koutroe@mail.amc.edu. Disclosures: The authors report no conflicts of interest or final disclosures. JCSO 2016;14(8):359-362. (22016 Frontline Medical Communications. doi: 10.12788/jicso.0223. 
TABLE Twenty-two adult cases of colonic leiomyosarcoma in the English literature of the post-GIST era (1995-2015)

\begin{tabular}{|c|c|c|c|c|c|c|c|c|}
\hline Study & Year & Country & Age, y & Gender & $\begin{array}{c}\text { Tumor } \\
\text { size, } \mathbf{c m}^{a}\end{array}$ & Site & LN & $\begin{array}{c}\text { Metastatic } \\
\text { disease }\end{array}$ \\
\hline Granero-Peiro ${ }^{4}$ & 2015 & Spain & 89 & $\mathrm{Fe}$ & 4.5 & Cecum & No & Liver \\
\hline Kono' & 2015 & Japan & 46 & $M$ & 12 & TR colon & No & Liver \\
\hline Yaren ${ }^{11}$ & 2014 & Turkey & 66 & $F$ & 4 & TR colon & No & No \\
\hline Samie $^{5}$ & 2013 & Germany & 65 & $M$ & NOS & Sigmoid colon & No & No \\
\hline Hamai $^{7}$ & 2012 & Japan & 66 & $\mathrm{~F}$ & 3 & Sigmoid colon & No & Liver, lung \\
\hline $\operatorname{Resch}^{12}$ & 2011 & Austria & 70 & $F$ & 3.7 & $\begin{array}{l}\text { Desc/sigmoid } \\
\text { colon }\end{array}$ & No & No \\
\hline $\operatorname{Resch}^{12}$ & 2011 & Austria & 56 & M & NOS & Cecum & No & Liver \\
\hline Agaimy $^{13}$ & 2007 & Germany & 72 & $\mathrm{~F}$ & 0.4 & Desc colon & No & No \\
\hline Agaimy $^{13}$ & 2007 & Germany & 52 & $M$ & NOS & Sigmoid colon & No & Liver \\
\hline Michalopoulos ${ }^{14}$ & 2004 & Greece & 67 & $\mathrm{~F}$ & 5.7 & TR colon & No & No \\
\hline Insabato ${ }^{15}$ & 2004 & Italy & 65 & M & 10 & Desc colon & No & Yes, NOS \\
\hline Miettinen $^{8}$ & 2000 & US/Finland & 54 & $M$ & 3.2 & Desc colon & NOS & NOS \\
\hline Miettinen $^{8}$ & 2000 & US/Finland & 61 & M & 4.2 & Asc colon & NOS & No \\
\hline Miettinen $^{8}$ & 2000 & US/Finland & 75 & $M$ & 6.5 & Asc colon & NOS & NOS \\
\hline Miettinen $^{8}$ & 2000 & US/Finland & 76 & $\mathrm{~F}$ & 7.8 & Cecum & NOS & NOS \\
\hline Miettinen $^{8}$ & 2000 & US/Finland & 36 & $\mathrm{~F}$ & 6.5 & Sigmoid colon & NOS & Lung \\
\hline Miettinen $^{8}$ & 2000 & US/Finland & 66 & M & NOS & Asc colon & NOS & Liver \\
\hline Miettinen $^{8}$ & 2000 & US/Finland & 41 & $M$ & 7.5 & Cecum & NOS & NOS \\
\hline Johnson ${ }^{16}$ & 1999 & US & 30 & $\mathrm{~F}$ & 3.5 & Desc colon & No & Liver \\
\hline Iwasa $^{17}$ & 1997 & Japan & 70 & $\mathrm{~F}$ & 10 & Asc colon & No & No \\
\hline Fallahzade $\mathrm{H}^{18}$ & 1995 & US & 63 & $\mathrm{~F}$ & NOS & Desc colon & Yes & No \\
\hline Fallahzade $\mathrm{H}^{18}$ & 1995 & US & 69 & $\mathrm{~F}$ & NOS & TR colon & No & No \\
\hline
\end{tabular}

Adj, adjuvant treatment; Asc, ascending; carbo, carboplatin; Desc, descending; doxo, doxorubicin; ifosf, ifosfamide; LN, lymph node involvement; mets, metastatic disease; NOS, not oth unknown

aLargest diameter.

agement, because chemotherapy and radiation therapy have shown zero to limited effect based on the existing literature. The patient did not opt for participation in a clinical trial. After her initial surgery, the patient was recovering relatively well and her wound was healing slowly, but uneventfully. However, about 4 months after her initial hospitalization, she presented again to the hospital, with severe abdominal pain. A repeat CT scan showed extensive peritoneal tumor with a large mass occupying the abdomen. The patient was not a candidate for any surgical intervention at that point. She died in the hospital a few days later while waiting nursing home placement.

\section{Discussion}

Primary leiomyosarcoma of the colon is an extremely rare neoplasm. We were able to retrieve only 22 adult cases published in the English literature in the past 20 years of the postGIST era (Table). We limited our research to the last 20 years, because definitive tools to differentiate leiomyosarcomas from GIST were not introduced until the late 1990s. ${ }^{3}$ The median age of the reported patients was 62 years (range, 30-89), and 12 out of $22(55 \%)$ were women. Presenting symptoms and endoscopic appearance leiomyosarcoma can be identical to other colonic neoplasms. The median largest diameter of the 


\begin{tabular}{|c|c|c|c|}
\hline Surgery & Chemotherapy & Relapse & Death \\
\hline Yes & Adj, NOS & No & No (unkn f-u) \\
\hline Yes & No & $x 2$ & No (unkn f-u) \\
\hline Yes & Adj (ifosf+doxo) & No & No (unkn f-u) \\
\hline Yes & No & No & No (unkn f-u) \\
\hline Yes & $\begin{array}{c}\text { Ifosf+epirubicin, } \\
\text { then } \\
\text { dacarbazine }\end{array}$ & x3 & Yes (in $58 \mathrm{mo}$ ) \\
\hline Yes & No & Yes & Yes (in $5 \mathrm{mo}$ ) \\
\hline $\begin{array}{c}\text { Yes, } \\
+ \text { RFA of liver } \\
\text { metastasis }\end{array}$ & $\begin{array}{l}\text { Ifosf+epirubicin, } \\
\text { then trabectedin, } \\
\text { then carbo + } \\
\text { VP-16 }\end{array}$ & $x 4$ & No (unkn f-u) \\
\hline $\begin{array}{l}\text { Endoscopically } \\
\text { resected }\end{array}$ & No & NOS & No (unkn f-u) \\
\hline Yes & No & NOS & No (unkn f-u) \\
\hline Yes & No & No & No (12 mo f-u) \\
\hline Yes & No & Yes & Yes (in $28 \mathrm{mo}$ ) \\
\hline Yes & NOS & NOS & Yes (in $37 \mathrm{mo}$ ) \\
\hline Yes & NOS & No & $\begin{array}{c}\text { No (f-u } 141 \\
\text { mo) }\end{array}$ \\
\hline Yes & NOS & NOS & Yes (in $6 \mathrm{mo}$ ) \\
\hline Yes & NOS & NOS & Yes (in $7 \mathrm{mo}$ ) \\
\hline Yes & NOS & Yes & Yes (in $38 \mathrm{mo}$ ) \\
\hline Yes & NOS & Yes & Yes (in $19 \mathrm{mo}$ ) \\
\hline Yes & NOS & NOS & $\begin{array}{c}\text { No (f-u } 185 \\
\text { mo) }\end{array}$ \\
\hline Yes +RT & Adj dacarbazine & $x 4$ & Yes (in $20 \mathrm{mo}$ ) \\
\hline Yes & No & No & No (f-u $12 \mathrm{mo}$ ) \\
\hline Yes & Yes & No & No (unkn f-u) \\
\hline Yes & No & No & No (unkn f-u) \\
\hline
\end{tabular}

nerwise specified; RFA, radiofrequency ablation; TR, transverse; unkn f-u, follow-up period

tumor among the 22 reported cases was $5.8 \mathrm{~cm}$. Four of the cases involved the cecum, 4 the ascending colon, 4 the transverse colon, 6 the descending colon, and 4 the sigmoid colon. Therefore, leiomyosarcoma seems to involve equally all the 5 parts of the colon. However, the small number of cases does not allow us to make certain conclusions.

The diagnosis of leiomyosarcoma is established based on histopathology and immunohistochemistry of the tissue specimen. An important criterion for diagnosis seems to be the histologic grade, which is determined by the number of mitotic figures per field. ${ }^{4}$ Unlike with GIST, c-Kit is nega-

tive in leiomyosarcoma and immunohistochemistry tests are positive for smooth muscle proteins such as actin, vimentin, and desmin, and negative for CD34, CD117, DOG1 (GIST markers) and s-100 protein (schwannoma marker). ${ }^{1}$ It is crucial that leiomyosarcoma is differentiated from GIST, because the treatment and prognosis is different.

The mainstay of treatment of colonic leiomyosarcoma remains surgical resection, even if disease is locally advanced. Despite adequate surgical resection, disease recurrence is frequent. Half of the reported cases relapsed, most of them multiple times ( 8 of 16 case relapsed; no data for 6). It is considered that colonic leiomyosarcoma rarely involves the lymph nodes. One patient of the 22 reported on in the literature, plus the patient we present here, had lymph node involvement. Liver is the organ most commonly affected by metastatic disease, although lung and bone metastases can occur. ${ }^{4}$ Among the 22 cases reported, 9 (41\%) record metastatic disease, of which 7 were in the liver and 2 in the lungs. In 1 case, there was questionable involvement of the spine. Despite frequent local recurrences and hematogenous spread, no effective adjuvant therapy has been established. ${ }^{5}$ Chemotherapy plays a limited role although it has been used by some centers. A 30\%-60\% clinical response rate of non-gastrointestinal soft-tissue sarcomas have been reported with combinations of docetaxel plus gemcitabine, or ifosfamide with anthracycline and/or dacarbazine. ${ }^{6,7}$ Response of colonic leiomyosarcomas to chemotherapy has not been systemically studied, and no effective adjuvant treatment approaches have been established because of the rarity of these neoplasms. In contrast to GISTs that respond well to tyrosine kinase inhibitors, leiomyosarcomas have not shown such response (c-KIT negative). ${ }^{1}$

The prognosis of colonic leiomyosarcoma is poor, with 9 of 22 reported patients (41\%) dying from their disease at a median of 24 months (range, 5-58). However, among those patients who survived, the time period of their follow-up is not well defined most of the time. Therefore, mortality may be even higher. Important prognostic features are the integrity of surgical resection and the malignant potential of the tumor. ${ }^{8}$ In 2000, Yeh and colleagues reported the outcomes of 40 patients with surgically resected rectal leiomyosarcoma (16 high grade, 23 low grade, 1 not specified). About half of the patients $(n=19)$ developed recurrence or metastasis postoperatively (median follow-up, 35 months). The overall and disease-free survival rates (1-year, 3-year, and 5-year) were $97 \%, 90 \%$, and $75 \%$ and $90 \%, 59 \%$, and $46 \%$, respectively. The investigators reported that histologic grade $(P=.037)$ and age divided by a level of 50 years $(P=$ $.009)$ were independently associated with disease-free survival, whereas gender, tumor size, tumor location, and operation type did not have any significant role. ${ }^{9} \mathrm{It}$ is reasonable to assume that rectal and colonic leiomyosarcomas behave similarly, although data on colonic leiomyosarcomas in the post-GIST era are scarce. Yamamoto and colleagues 


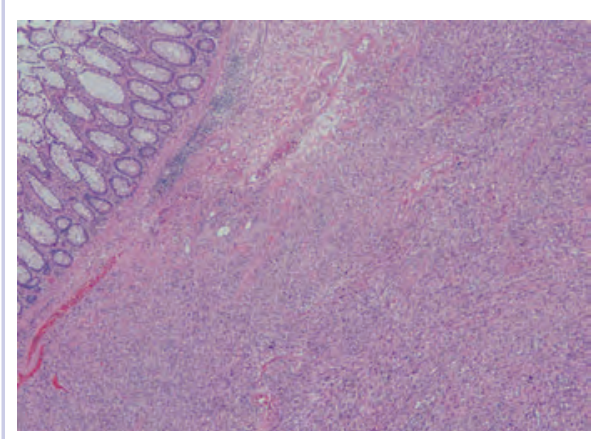

FIGURE 1 The unremarkable colonic mucosa is to the left of the field, and the tumor replaces the normal muscularis propria on the right. The tumor has a fascicular pattern of architecture with spindled tumor cells intersecting each other at right angles. Even at this low power, the atypia of the fumor is evident with scattered large, hyperchromatic cells. Mitotic figures are also evident. (40x total magnification)

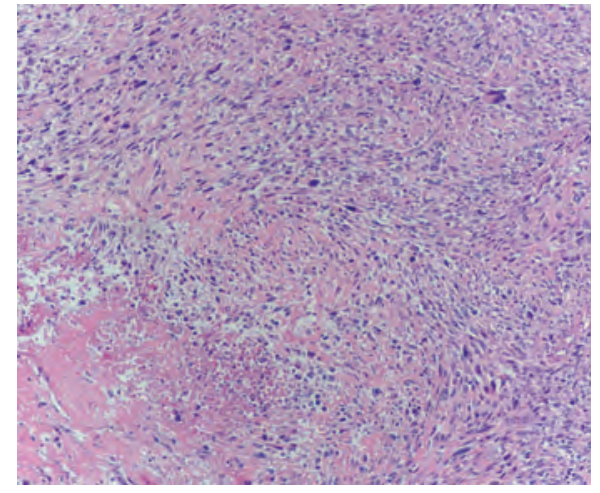

FIGURE 2 At 100x total magnification, there are spindle cells with pronounced atypia, including hyperchromasia and pleomorphism. Nuclei are cigar-shaped with blunt ends, and there are scattered cytoplasmic vacuoles. Cytoplasm is eosinophilic and fibrillary. There is also tumor necrosis within the lower left field.

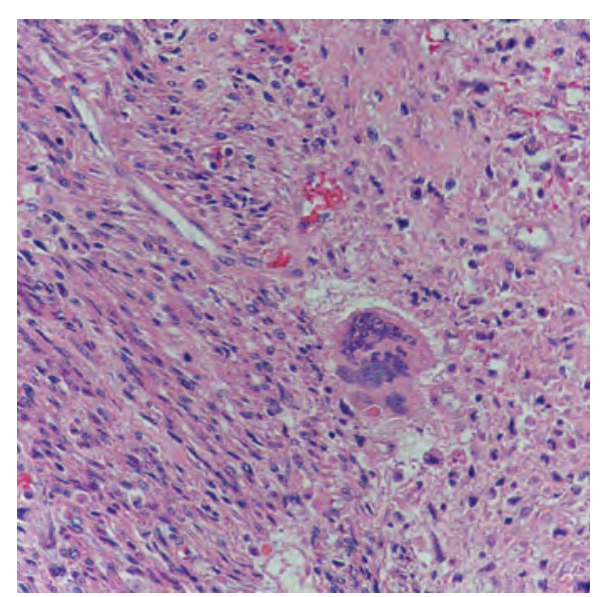

FIGURE 3 At 200x total magnification, there are scattered osteoclast-like giant cells. Background cellular atypia, including hyperchromasia and pleomorphism, is evident. recently reviewed 55 cases of gastrointestinal leiomyosarcomas from the post-GIST era, and the estimated 5-year overall survival rate was $51.6 \%$ among 29 of the patients with long-term follow-up. The strongest prognostic factor was tumor size, with tumors of $\geq 5 \mathrm{~cm}$ correlating with shorter survival $(P=.0016)$. Mitotic rate and primary site of the tumor did not seem to have any prognostic value. ${ }^{10}$

The patient we present in this report has similar characteristics with the published patients with high-grade leiomyosarcoma, with the exception of lymph node involvement,

\section{References}

1. Kono M, Tsuji N, Ozaki N, et al. Primary leiomyosarcoma of the colon. Clin J Gastroenterol. 2015;8:217-222.

2. Hirota S, Isozaki K, Moriyama Y, et al. Gain-of-function mutations of c-kit in human gastrointestinal stromal tumors. Science. 1998;279:577-580

3. Aggarwal G1, Sharma S, Zheng M, et al. Primary leiomyosarcomas of the gastrointestinal tract in the post-gastrointestinal stromal tumor era. Ann Diagn Pathol. 2012;16:532-540.

4. Granero-Peiró L, Martínez-Ortega P, Sánchez-Justicia C, et al. Leiomyosarcoma of the ascending colon: a rare tumor with poor prognosis. Rev Esp Enferm Dig. 2015;107:580-581.

5. Abdel Samie A, Sun R, Fayyazi A, et al. Leiomyosarcoma of the sigmoid colon: a rare cause of intestinal intussusception. J Gastrointest Cancer. 2014;45 (suppl 1):6-9.

6. Hensley ML, Maki R, Venkatraman E, et al. Gemcitabine and docetaxel in patients with unresectable leiomyosarcoma: results of a phase II trial. J Clin Oncol. 2002;20:2824-2831.

7. Hamai Y, Hihara J, Emi M, et al. Leiomyosarcoma of the sigmoid colon with multiple liver metastases and gastric cancer: a case report. BMC Gastroenterol. 2012;12:98.

8. Miettinen M, Sarlomo-Rikala M, Sobin LH, et al. Gastrointestinal stromal tumors and leiomyosarcomas in the colon: a clinicopathologic, immunohistochemical, and molecular genetic study of 44 cases. Am J Surg Pathol. 2000;24:1339-1352.

9. Yeh CY, Chen HH, Tang R, et al. Surgical outcome after curative resection of rectal leiomyosarcoma. Dis Colon Rectum. 2000;43:1517-1521.

10. Yamamoto H, Handa M, Tobo T, et al. Clinicopathological features which is very rare. The outcome for our patient was poor after treatment with only surgical resection, with the tumor spreading and the patient dying 4 months after diagnosis.

In conclusion, leiomyosarcoma of the colon is a rare neoplasm with poor prognosis. The differentiation from GIST is based on histopathology and immunohistochemistry and is important because the treatment and prognosis is different. Surgery remains the mainstay of leiomyosarcoma treatment. Despite the high relapse and hematogenous spread rates, no adjuvant therapy has been established. of primary leiomyosarcoma of the gastrointestinal tract following recognition of gastrointestinal stromal tumours. Histopathology. 2013;63:194-207

11. Yaren A, Değirmencioğlu S, Callı Demirkan N, et al. Primary mesenchymal tumors of the colon: a report of three cases. Turk J Gastroenterol. 2014;25:314-318.

12. Resch T, Oberhuber R, Zitt M, et al. Leiomyosarcoma of the colon: unresolved issues of a rare but highly aggressive malignancy. Am Surg. 2011;77:E62-64.

13. Agaimy A, Wünsch PH. True smooth muscle neoplasms of the gastrointestinal tract: morphological spectrum and classification in a series of 85 cases from a single institute. Langenbecks Arch Surg. 2007;392:75-81.

14. Michalopoulos A, Papadopoulos VN, Basdanis G, et al. Colorectal gastrointestinal mesenchymal tumours. Report of a stromal case of the rectum (GIST) and a leiomyosarcoma of the transverse colon. Tech Coloproctol. 2004;8(suppl 1):s155-157.

15. Insabato L, Di Vizio D, Ciancia G, et al. Malignant gastrointestinal leiomyosarcoma and gastrointestinal stromal tumor with prominent osteoclast-like giant cells. Arch Pathol Lab Med. 2004;128:440-443.

16. Johnson MA, Gibbs DH, Gouldman J, et al. Leiomyosarcoma of the colon: a second malignant neoplasm after treatment for a Wilms' tumor. Am Surg. 1999;65:6-10.

17. Iwasa K, Taniguchi K, Noguchi M, et al. Leiomyosarcoma of the colon presenting as acute suppurative peritonitis. Surg Today. 1997;27:337-344.

18. Fallahzadeh H. Leiomyosarcoma of colon: report of two cases. Am Surg. 1995;61:294-296. 